\title{
Rapid detection of FGFR3 gene mutation in achondroplasia by DHPLC system-coupling heteroduplex and fluorescence-enhanced primer-extension analysis
}

Received: 1 March 2004 / Accepted: 21 April 2004/Published online: 18 June 2004

(C) The Japan Society of Human Genetics and Springer-Verlag 2004

\begin{abstract}
Achondroplasia is a common form of human dwarfism with characteristically rhizomelic shortening of extremities and relative macrocephaly. It is transmitted as an autosomally dominant inheritance, and about $80 \%$ of affected individuals result from sporadic mutations without positive family histories. Achondroplasia comes from the genetic point mutations in the fibroblastic growth factor receptor 3 gene (FGFR3), which enables abnormal cartilage growth-plate differentiation and insufficient bony development. The most common genetic mutations in this receptor are $\mathrm{G}$ to $\mathrm{A}$ at position 1138 (G1138A), which result in the substitution of glycine to arginine at codon 380 . Based on genetic information, molecular genetic testing can provide an exact diagnosis comparing to radiological and prenatal ultrasound evaluations. Here we introduce denaturing highperformance liquid chromatography (DHPLC) for the detection of 17 cases of achondroplasia and 120 unaffected cases. After coupling heteroduplex and fluores-
\end{abstract}

Y.-N. Su • Y.-H. Chien

Department of Medical Genetics,

National Taiwan University Hospital,

National Taiwan University, Taipei,

Taiwan, ROC

C.-N. Lee · S.-C. Chien · C.-A. Chen $(\varangle)$

Department of Obstetrics and Gynecology,

National Taiwan University Hospital,

College of Medicine, National Taiwan University,

No.7, Chung-Shan South Road,

Taipei, Taiwan, ROC

E-mail: cachen@ntumc.org

C.-C. Hung

Institute of Biomedical Engineering,

National Taiwan University, Taipei,

Taiwan, ROC

Y.-H. Chien

Department of Pediatrics,

National Taiwan University Hospital,

College of Medicine, National Taiwan University,

Taipei, Taiwan, ROC cence-enhanced primer-extension analysis, all affected patients with G1138A were identified successfully. In conclusion, we demonstrated that DHPLC is an efficient, accurate, and sensitive technique to detect the single gene mutation of achondroplasia in clinical applications.

Keywords Achondroplasia - Fibroblast growth factor receptor 3 gene $\cdot$ Denaturing high-performance liquid chromatography · Heteroduplex analysis · Primer-extension analysis

\section{Introduction}

Achondroplasia (OMIM 100800) is the most common form of short-limbed dwarfism in human beings, and its estimated prevalence rate is approximately between $1 / 15,000$ and 1/70,000 (Bellus et al. 1994). The characteristic presentations of achondroplasia include proximal shortening of the extremities, genu varum, trident hand, limiting of elbow extension, exaggerating lumbar lordosis, megalencephaly, and the face with frontal bossing and midface hypoplasia (Hunter et al. 1998).

Achondroplasia is inherited in an autosomally dominant manner with full penetrance. Over $80 \%$ of affected individuals are born to parents of average height, and most come from sporadic mutations (Murdoch et al. 1970). Normal parents have a low risk for recurrence of giving birth to another child with achondroplasia. Couples with one affected individual may carry a $50 \%$ risk of transmitting the mutant gene to each child with achondroplasia. A severe lethal form, homozygous achondroplasia, may carry about a $25 \%$ risk in a family whose parents are both achondroplasia (Castiglia 1996), and the affected infants often die in their first year from severe neurological deficits or respiratory failure.

Achondroplasia is a specific mutation at a fibroblastic growth factor receptor 3 (FGFR3) 
transmembrane domain on chromosomal locus $4 \mathrm{p} 16.3$ (Francomano et al. 1994; Le Merrer et al. 1994; Velinov et al. 1994). Two different point mutations in the FGFR3 will be detected in more than $99 \%$ of achondroplasia resulting in a glycine-to-arginine substitution at codon 380 (G380R). Among mutations, more than $97 \%$ of patients are attributable to the $G$ to A point mutation at nucleotide 1138 of the FGFR3 gene (G1138A), and only $1 \%$ of achondroplasia has a $\mathrm{G}$ to $\mathrm{C}$ point mutation at the same site (G1138C) (Bellus et al. 1995; Rousseau et al. 1994; Shiang et al. 1994). The guanosine 1138 nucleotide has been described as the most mutable nucleotide in the human genome (Bellus et al. 1995). Missense mutations in the transmembrane domain the of FGFR3 gene result in constitutive activation of the receptor, which may give rise to inappropriate cartilage growth-plate differentiation and abnormal long-bone development (Webster and Donoghue 1996).

Denaturing high-performance liquid chromatography (DHPLC) has been clinically used in molecular diagnosis of several genetic diseases, and it automatically can detect the single-base substitution, small insertion, and deletions. The sensitivity and the specificity of DHPLC have been reported from 96 to $100 \%$ in range (Jones et al. 1999; O'Donovan et al. 1998). When DHPLC is combinating with heteroduplex analysis, it takes 8 min per sample to distinguish the heteroduplex profiles from homoduplices. However, it is dependent on the configuration of the instrument (Cotton 1997; Underhill et al. 1997).

Primer-extension is a versatile method, and detecting extension products with a variety of techniques have been proposed. When the primer-extension products are analyzed by the DHPLC system, the advantages will be an automated sample loading and analysis, shortened analytic time, and no further purification steps are needed (Su et al. 2003). Furthermore, the WAVE Optimized HS Staining Solution I in conjunction with the Transgenomic FDX fluorescence detector may provide a higher sensitivity in detection of the primer-extension analyses. In this study, we used DHPLC to combine heteroduplex analysis with fluorescence-enhanced primer-extension analysis to genotype the FGFR3 gene. This will be a powerful, efficient, and accurate analytical method in diagnosing achondroplasia.

\section{Materials and methods}

\section{Sample preparation}

After completing genetic counseling and obtaining informed consent for participation, a $5-10 \mathrm{ml}$ blood sample from each individual was drawn. All DNA samples were extracted from peripheral whole blood by using a Puregene DNA Isolation Kit (Gentra Systems, Inc., Minneapolis, MN, USA).
Primer design and PCR for heteroduplex analysis by DHPLC

PCR for the fragments was conducted according to a modified protocol as originally described by Shiang et al. (1994). To detect the $1138 \mathrm{G}$ to A point mutation in the FGFR3 gene, the sequences of these primers are $5^{\prime}$ AGGAGCTGGTGGAGGCTGA- $3^{\prime}$ and 5'-GGAGATCTTGTGCACGGTGG-3'. The PCR products spanning exon 10 of FGFR3 gene were used herein and generate a fragment of $164 \mathrm{bp}$. More than $99 \%$ of achondroplasia could be detected in this amplicon.

PCR techniques for the provided DNA fragments were performed in a total volume of $25 \mu \mathrm{l}$ containing $100 \mathrm{ng}$ of genomic DNA, $0.12 \mu \mathrm{M}$ of each primer, $100 \mu \mathrm{M}$ dNTPs, $10 \mathrm{mM}$ Tris- $\mathrm{HCl}(\mathrm{pH} 8.3), 50 \mathrm{mM}$ $\mathrm{KCl}, 2 \mathrm{mM} \mathrm{MgCl}_{2}$, and $0.5 \mathrm{U}$ of AmpliTaq Gold enzyme (PE Applied Biosystems, Foster City, CA, USA). Amplification was performed in a multiblock system (MBS) thermocycler (ThermoHybaid, Ashford, UK). PCR amplification was initiated with a denatured step at $95^{\circ} \mathrm{C}$ for $10 \mathrm{~min}$ followed by 35 cycles consisting of denaturizing at $94^{\circ} \mathrm{C}$ for $30 \mathrm{~s}$, annealing at $65^{\circ} \mathrm{C}$ for $30 \mathrm{~s}$, and extension at $72^{\circ} \mathrm{C}$ for $30 \mathrm{~s}$ and then a final extension step at $72^{\circ} \mathrm{C}$ for $10 \mathrm{~min}$.

Preparation of PCR products for primer-extension (minisequencing) reaction

For primer-extension analysis, PCR products, which were used for heteroduplex analysis, were treated with GFX PCR DNA and Gel Band Purification Kit (Amersham Biosciences, Piscataway, NJ, USA) to remove unincorporated primers and dNTPs, then the purified PCR products were subsequently used for the primer-extension reactions. The level of PCR products were quantified by using a GeneQuant pro spectrophotometer (Amersham Pharmacia Biotech, Piscataway, NJ, USA) with samples being diluted to a final concentration of $10 \mathrm{ng} / \mu \mathrm{l}$.

Minisequencing primers design and primer-extension reactions for DHPLC

The sequence of the minisequencing extension primer used in this study to detect the G1138A mutation was 5'-AGGAGCTGGTGGAGGCTGA-3'. Primer-extension reactions were carried out in a volume of $20 \mu$, the sample containing $50 \mathrm{ng}$ purified input PCR product and $0.5 \mu \mathrm{M}$ minisequencing primer, $25 \mu \mathrm{M}$ of each four unlabeled ddNTPs (Amersham Pharmacia Biotech, Piscataway, NJ, USA), $0.5 \mathrm{U}$ of ThermoSequenase DNA polymerase (Amersham Biosciences, Piscataway, NJ, USA), and $2 \mu \mathrm{l}$ of $10 \times$ reaction buffer provided by the manufacturer. The reaction was carried out in a MBS thermocycler (ThermoHybaid, Ashford, UK) with an initial denaturation step at $96^{\circ} \mathrm{C}$ for $60 \mathrm{~s}$ followed by 
50 cycles of $96^{\circ} \mathrm{C}$ for $15 \mathrm{~s}, 43^{\circ} \mathrm{C}$ for $15 \mathrm{~s}, 60^{\circ} \mathrm{C}$ for $100 \mathrm{~s}$, and finally $96^{\circ} \mathrm{C}$ for $30 \mathrm{~s}$.

\section{DHPLC analysis}

The DHPLC system used for analyzing heteroduplexes was a Transgenomic Wave Nucleic Acid Fragment Analysis System (Transgenomic, Inc., San Jose, CA, USA). DHPLC was carried out on automated HPLC instrument equipped with a DNASep column (Transgenomic, Inc., San Jose, CA, USA). DHPLC-grade acetonitrile (9017-03, JT Baker, Phillipsburg, NJ, USA) and triethylammonium acetate (TEAA, Transgenomic, Inc., Crewe, UK) were used to constitute the mobile phase. The mobile phases comprised $0.05 \%$ acetonitrile in $0.1 \mathrm{M}$ TEAA (eluent $\mathrm{A}$ ) and $25 \%$ acetonitrile in 0.1 M TEAA (eluent B).

For heteroduplex detection, crude PCR products subjected to an additional 10 -min $95^{\circ} \mathrm{C}$ denaturing step followed by gradual reannealing from 95 to $65^{\circ} \mathrm{C}$ over a period of $30 \mathrm{~min}$ prior to analysis were eluted at a flow rate of $0.9 \mathrm{ml} / \mathrm{min}$. Start points and end points of the gradient (in eluent B) were 47 and $56 \%$ in $4.5 \mathrm{~min}$. The temperature for successful resolution of heteroduplex molecules was $66^{\circ} \mathrm{C}$, which were all adjusted by using an algorithm provided by the WAVEmaker system control software version 4.1.42 (Transgenomic, Inc., San Jose, CA, USA). For analyzing, $8 \mu \mathrm{l}$ of PCR product were injected in each running. Heterozygous profiles were identified by visual inspection of the chromatograms on the basis of the appearance of additional earlier eluting peaks.

For primer-extension analysis, $8 \mu \mathrm{l}$ of primer-extension reaction product was loaded on a DNASep column. DHPLC was performed with a column temperature of $75^{\circ} \mathrm{C}$ with a flow rate of $0.9 \mathrm{ml} / \mathrm{min}$. For detecting primer-extension analyses, the UV detector set with $260 \mathrm{~nm}$ and the WAVE Optimized HS Staining Solution I (Transgenomic, Inc., San Jose, CA, USA) conjuncts with the Transgenomic FDX fluorescence detector (Transgenomic, Inc., San Jose, CA, USA) were used. Start points and end points of the gradient (expressed in eluent $\mathrm{B} \%$ ) were 21 and $35 \%$ in $4.5 \mathrm{~min}$.

Sequence analysis

For sequencing, PCR products were purified by solidphase extraction and bidirectionally sequenced with the Applied Biosystems Taq DyeDeoxy terminator cycle sequencing kit (Applied Biosystems) according to the manufacturer's instructions. Sequencing reactions were separated on an Applied Biosystems 3100 sequencer.

\section{Results}

A total of 17 patients with achondroplasia and 120 healthy controls, including family members of the affected cases, were tested by using heteroduplex and primer-extension analysis by DHPLC. All sequence variants with G1138A had been previously confirmed as exhibiting variations in the FGFR3 gene by analyses of restriction enzyme digestion and sequence analysis. By heteroduplex analysis, all 17 variants from the homozygous reference wild-type DNA were identified successfully. DHPLC chromatograms of the common mutation G1138A could be differentiated successfully from the homozygous counterpart. The results that were analyzed by DHPLC were all further confirmed by direct sequencing with $100 \%$ sensitivity and specificity (Fig. 1).

Moreover, to identify and confirm the specific sequence variant of G1138A, we used DHPLC analysis of the primer-extension reactions for genotyping
Fig. 1a, b Denaturing highperformance liquid chromatography (DHPLC) and sequence analysis of a patient with G1138A in the FGFR3 gene compared to wild type. a Chromatography and sequence analysis indicate the wild-type DNA. b Chromatography and sequence analysis indicate the patient with G1138A in the FGFR3 gene (a)

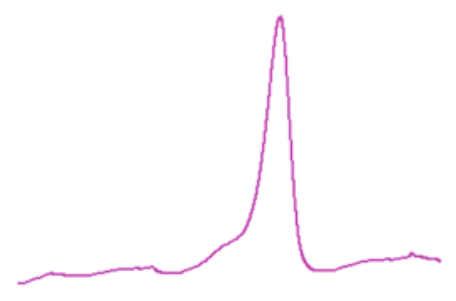

(b)

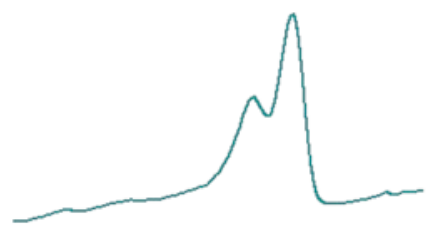

\section{c. $1138 \mathrm{G}>\mathrm{A}$}

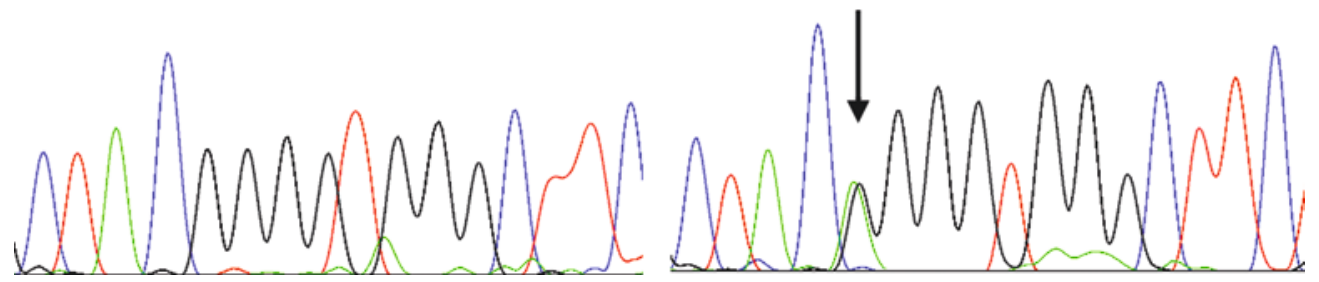


FGFR3 mutations. DHPLC analysis of the primerextension reactions detected by the UV detector and the fluorescence detector both resulted in reproducible chromatograms that could be used to more easily determine the genotype at the polymorphic site. There were two extended peaks that could be identified for heterozygous individuals, while only one single extended peak was able to be detected for wild-type homozygous counterparts. In our study, by using the fluorescence detector, signal intensity and detection ability could further be improved about ten times when compared with traditional UV detection (Fig. 2).

\section{Discussion}

The diagnosis of achondroplasia usually relies on a combination of clinical evaluation and radiographic features until the development of molecular diagnosis, which is based on a nearly single-gene mutation. Shiang et al. developed the PCR-RFLP method to screen for the occurrence of the G380R mutation in FGFR3 (Shiang et al. 1994). Recently, direct DNA sequencing has made the diagnosis in a more sensitive and corrective way. Such molecular genetic testing is helpful in diagnosing atypical individuals who may be asymptomatic or minimally affected, and for distinguishing sporadic or familial events.

Although direct DNA sequencing is the gold standard for genetic testing in the modern era, major disadvantages that restrict the use of direct sequencing as a screening program include the facts that it is expensive, time-consuming, produces annoying background noise, and is technique-dependent. Recently, DHPLC has become a novel, high-capacity technique for analysis of the human genome. Its clinical uses in a wide range of genetic diseases for mutational detection has been reported (Bunn et al. 2002; Ravnik-Glavac et al. 2002). By using heteroduplex analysis in our study, DHPLC chromatograms revealed all different heterozygous mutations with the characteristic elution profiles that could be markedly distinguished from homozygous wild-type DNA. The distinct elution profiles let us easy identify the genetic mutation in achondroplasia. Furthermore, we introduced the primer-extension analysis coupled with the DHPLC system as a valuable tool to genotype FGFR3
Fig. 2a-f Denaturing highperformance liquid

chromatography (DHPLC) chromatograms from mutant and wild-type subjects detected by primer-extension analysis. a,b,c Elution profiles of primerextension analysis for primer only, wild-type, and mutant subjects by UV detector, respectively. d,e,f Elution profiles of primer-extension analysis for primer only, wild-type, and mutant subjects by WAVE Optimized HS Staining Solution I system in conjunction with the Transgenomic FDX fluorescence detector, respectively. Down arrow denotes the peak for primer only. Asterisk denotes the peak for the primer-extension product with primer adds ddG. Dash denotes the peak for the primer-extension product (primer adds ddA)
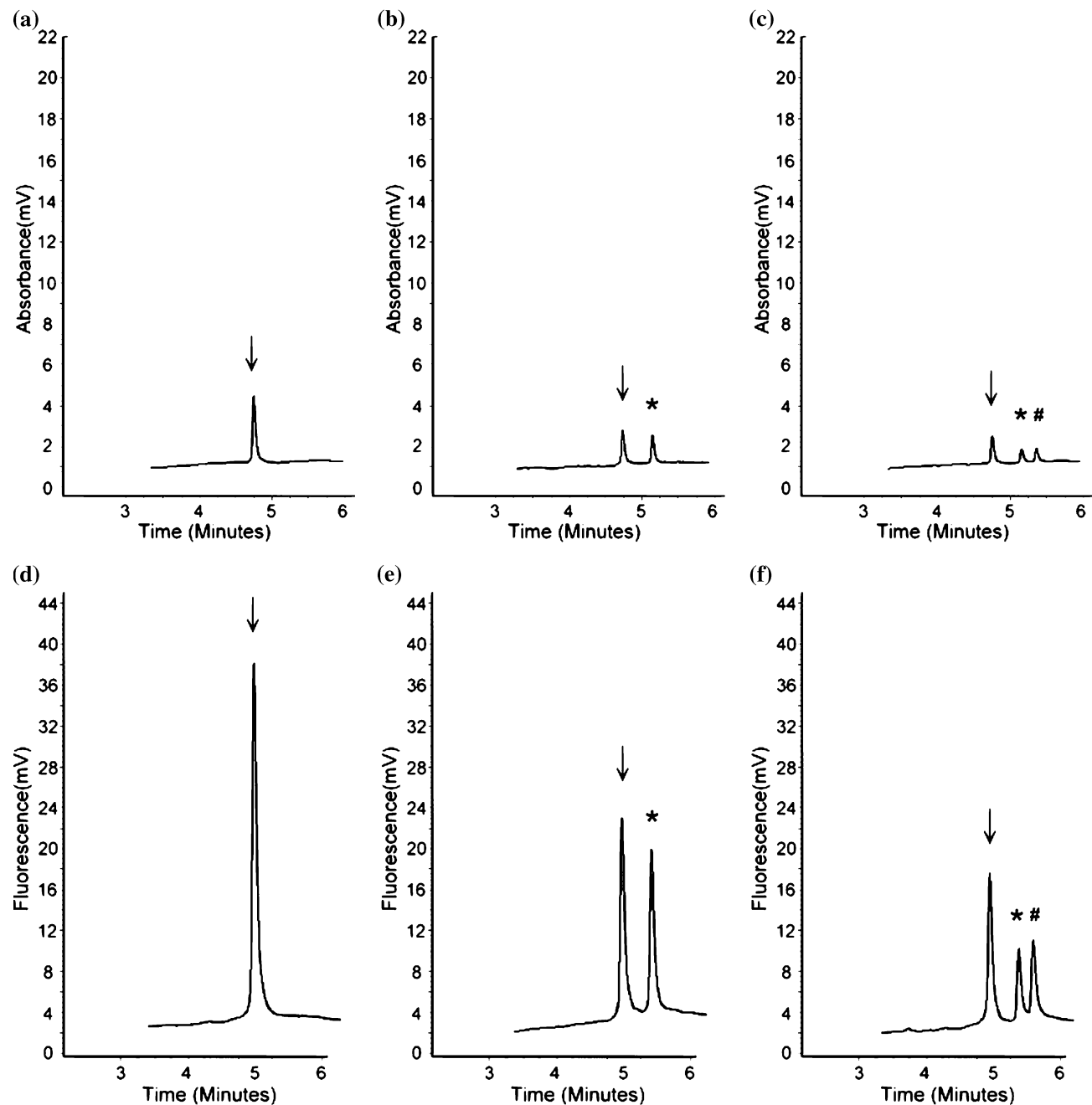

(e)

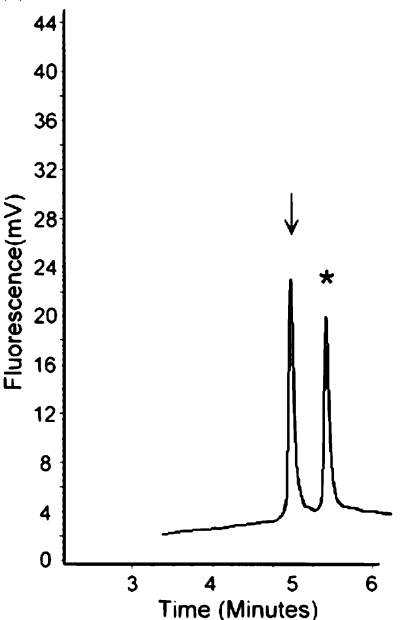

(f)

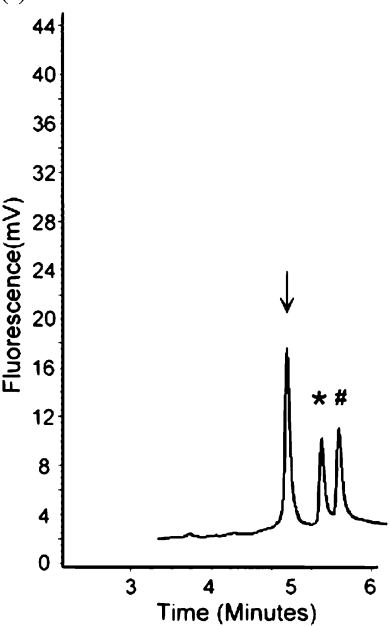


mutations in achondroplasia. After mixing up a minisequencing primer with the extended ddNTP, the chromatogram from primer-extension products showed a single extended peak detected from wild-type homozygous individuals and two extended peaks for heterozygous ones. The first peak, corresponding to the unextended minisequencing primer, always presents the same retention time in each chromatogram. The result was associated with the hydrophobicity of the given oligonucleotide primer and the number of electronic interactions between negatively charged phosphate groups on the DNA backbones and TEAA. Compared with direct sequencing, this is a cheaper and more timesaving method. Due to automated sample loading and analysis, brief analytic time and no further purification steps after the primer-extension are required; the method we described above provides an easier method of analysis. To enhance the detection of sensitivity for the minisequencing extended products, we applied the newly developed WAVE Optimized HS Staining Solution I system in conjunction with the Transgenomic FDX fluorescence detector. In our setting, by using the fluorescence detector, we got at least a ten-times higher detection signal and less background noise comparet with the traditional UV detector.

Although more than $97 \%$ of patients with achondroplasia are attributed to G1138A mutation, there are still other mutations in the FGFR3 gene have been identified. Moreover, there are several similar phenotypes associated with mutations in the FGFR3 gene other than G1138A such as hypochondroplasia, thanatophoric dysplasia, several achondroplasia with development delay, and acanthosis nigrican (PassosBueno et al. 1999). For other mutations in FGFR3 gene, we developed the detection protocol based on coupling the heteroduplex analysis by DHPLC and direct sequencing (data not shown).

In summary, by using the combination of heteroduplex and primer-extention analysis, especially further application of the fluorescence-enhanced detection system, DHPLC serves as a highly accurate, rapid, and efficient method for detecting the common G1138A mutation.

Acknowledgements This work was supported by grants from the Department of Health, Executive Yuan, Taiwan, ROC (DOH92TD-1059).

\section{References}

Bellus GA, Escallon CS, Ortiz de Luna R, Shumway JB, Blakemore KJ, McIntosh I, Francomano CA (1994) First-trimester prenatal diagnosis in couple at risk for homozygous achondroplasia. Lancet 344:1511-1512
Bellus GA, Hefferon TW, Ortiz de Luna RI, Hecht JT, Horton WA, Machado M, Kaitila I, McIntosh I, Francomano CA (1995) Achondroplasia is defined by recurrent G380R mutations of FGFR3. Am J Hum Genet 56:368-373

Bunn CF, Lintott CJ, Scott RS, George PM (2002) Comparison of SSCP and DHPLC for the detection of LDLR mutations in a New Zealand cohort. Hum Mutat 19:311

Castiglia PT (1996) Achondroplasia. J Pediatr Health Care 10:180182

Cotton RG (1997) Slowly but surely towards better scanning for mutations. Trends Genet 13:43-46

Francomano CA, Ortiz de Luna RI, Hefferon TW, Bellus GA, Turner CE, Taylor E, Meyers DA, Blanton SH, Murray JC, McIntosh I et al (1994) Localization of the achondroplasia gene to the distal $2.5 \mathrm{Mb}$ of human chromosome $4 \mathrm{p}$. Hum Mol Genet 3:787-792

Hunter AG, Bankier A, Rogers JG, Sillence D, Scott CI Jr (1998) Medical complications of achondroplasia: a multicentre patient review. J Med Genet 35:705-712

Jones AC, Austin J, Hansen N, Hoogendoorn B, Oefner PJ, Cheadle JP, O'Donovan MC (1999) Optimal temperature selection for mutation detection by denaturing HPLC and comparison to single-stranded conformation polymorphism and heteroduplex analysis. Clin Chem 45:1133-1140

Le Merrer M, Rousseau F, Legeai-Mallet L, Landais JC, Pelet A, Bonaventure J, Sanak M, Weissenbach J, Stoll C, Munnich A et al (1994) A gene for achondroplasia-hypochondroplasia maps to chromosome 4p. Nat Genet 6:318-321

Murdoch JL, Walker BA, Hall JG, Abbey H, Smith KK, McKusick VA (1970) Achondroplasia - a genetic and statistical survey. Ann Hum Genet 33:227-244

O’Donovan MC, Oefner PJ, Roberts SC, Austin J, Hoogendoorn B, Guy C, Speight G, Upadhyaya M, Sommer SS, McGuffin P (1998) Blind analysis of denaturing high-performance liquid chromatography as a tool for mutation detection. Genomics 52:44-49

Passos-Bueno MR, Wilcox WR, Jabs EW, Sertie AL, Alonso LG, Kitoh H (1999) Clinical spectrum of fibroblast growth factor receptor mutations. Hum Mutat 14:115-125

Ravnik-Glavac M, Atkinson A, Glavac D, Dean M (2002) DHPLC screening of cystic fibrosis gene mutations. Hum Mutat 19:374-383

Rousseau F, Bonaventure J, Legeai-Mallet L, Pelet A, Rozet JM, Maroteaux P, Le Merrer M, Munnich A (1994) Mutations in the gene encoding fibroblast growth factor receptor-3 in achondroplasia. Nature 371:252-254

Shiang R, Thompson LM, Zhu YZ, Church DM, Fielder TJ, Bocian M, Winokur ST, Wasmuth JJ (1994) Mutations in the transmembrane domain of FGFR3 cause the most common genetic form of dwarfism, achondroplasia. Cell 78:335-342

$\mathrm{Su}$ YN, Lee CN, Hung CC, Chen CA, Cheng WF, Tsao PN, Yu CL, Hsieh FJ (2003) Rapid detection of beta-globin gene (HBB) mutations coupling heteroduplex and primer-extension analysis by DHPLC. Hum Mutat 22:326-336

Underhill PA, Jin L, Lin AA, Mehdi SQ, Jenkins T, Vollrath D, Davis RW, Cavalli-Sforza LL, Oefner PJ (1997) Detection of numerous Y chromosome biallelic polymorphisms by denaturing high-performance liquid chromatography. Genome Res 7:996-1005

Velinov M, Slaugenhaupt SA, Stoilov I, Scott CI Jr, Gusella JF, Tsipouras P (1994) The gene for achondroplasia maps to the telomeric region of chromosome 4p. Nat Genet 6:314-317

Webster MK, Donoghue DJ (1996) Constitutive activation of fibroblast growth factor receptor 3 by the transmembrane domain point mutation found in achondroplasia. EMBO J 15:520-527 